\title{
PUBLICACIÓN Y FACTORES ASOCIADOS EN DOCENTES UNIVERSITARIOS DE INVESTIGACIÓN CIENTIIFICA DE ESCUELAS DE MEDICINA DEL PERÚ
}

\author{
Reneé Pereyra-Elías ${ }^{1, a}$, Juan Jesús Huaccho-Rojas²,a, Álvaro Taype-Rondan²,b, \\ Christian R. Mejia ${ }^{1, b}$, Percy Mayta-Tristán ${ }^{1, b}$
}

\begin{abstract}
RESUMEN
Objetivos. Evaluar la frecuencia de publicación y sus factores asociados en docentes universitarios de investigación científica de escuelas de Medicina del Perú. Materiales y métodos. Estudio de corte transversal analítico. Se incluyó a todos los docentes universitarios de los cursos de investigación de las 32 escuelas de medicina del Perú en el año 2011. La búsqueda de publicaciones se realizó mediante Google Scholar, SCOPUS y Medline. Se calculó las razones de prevalencias crudas y ajustadas (RPa) con intervalos de confianza al 95\% mediante regresión de Poisson simple y múltiple con varianza robusta. Resultados. De los 201 docentes universitarios, 43,8\% nunca ha publicado un artículo en una revista, $26,9 \%$ publicó un artículo original en una revista indizada en Medline y $16,4 \%$ lo hizo en los últimos dos años. Solo $3,0 \%$ han sido autores corresponsales en alguna revista indizada no peruana. Los factores asociados con haber publicado un artículo original en Medline durante los dos últimos años es ser menor de 40 años de edad (RPa: 2,97; IC 95\%:1,21-7,32), ser profesor en una universidad donde se requiere tesis obligatoria para graduarse (RPa: 8,84; IC 95\%: 2,60-30,12) y trabajar para una universidad altamente productiva (RPa: 3,24; IC 95\%: 1,03-10,20). Conclusiones. La frecuencia de publicación de los docentes en investigación de las escuelas de medicina del Perú es baja. Los docentes universitarios jóvenes y los que trabajan para universidades científicamente productivas presentaron más probabilidades de publicar en una revista indizada en Medline.
\end{abstract}

Palabras clave: Educación médica; Docentes; Universidades; Publicaciones; Investigadores; Perú (fuente: DeCS BIREME).

\section{PUBLISHING AND ITS ASSOCIATED FACTORS IN TEACHERS OF SCIENTIFIC RESEARCH IN SCHOOLS OF MEDICINE IN PERU}

\begin{abstract}
Objectives. To evaluate the frequency of publication and its associated factors by professors of scientific research in medical schools in Peru. Materials and methods. This was a cross-sectional study. We included all teachers of research courses from the 32 medical schools in Peru in 2011. The publication search was conducted using Google Scholar, Scopus and Medline. Both the crude and adjusted prevalence ratios (aPR) were calculated with confidence intervals at $95 \%$ using simple and multiple Poisson regression with robust variance. Results. Of the 201 university teachers, $43.8 \%$ had never published an article in a journal, $26.9 \%$ had an original article published in a journal indexed in Medline and $16.4 \%$ did so in the past two years. Only $3 \%$ had been corresponding authors in non-Peruvian, indexed journals Factors associated with having an original article published in Medline in the past two years were: being under 40 years of age (aPR 2.97, 95\% Cl: 1.21-7.32), being a professor at a university where a final thesis is required for graduation (aPR 8.84, 95\% Cl: 2.60-30.12) and working for a highly productive university (aPR 3.24, 95\% Cl: 1.03-10.20). Conclusions. The frequency of publication of research faculty in medical schools in Peru is low. Young university teachers and those working at scientifically productive universities were more likely to publish in an indexed journal.
\end{abstract}

Key words: Education, medical; Faculty; Universities; Publications; Research personnel; Peru (source: MeSH, NLM).

Escuela de Medicina, Universidad Peruana de Ciencias Aplicadas. Lima, Perú.

Facultad de Medicina, Universidad de San Martín de Porres. Lima, Perú.

a Estudiante de Medicina; ${ }^{b}$ médico cirujano

Recibido: : 24-06-14 Aprobado: 03-09-14

Citar como: Pereyra-Elías R, Huaccho-Rojas JJ, Taype-Roldan A, Mejia CR, Mayta-Tristán P. Publicación y factores asociados en docentes universitarios de investigación científica de escuelas de medicina del Perú. Rev Peru Med Exp Salud Publica. 2014;31(3):424-30. 


\section{INTRODUCCIÓN}

La investigación científica está estrechamente relacionada con el desarrollo social y económico de un país o una región ${ }^{(1,2)}$, y es cuantificada a través de los artículos publicados en revistas indizadas y patentes (3). Las universidades son el espacio por excelencia para la generación de investigación, ya que su función principal es la generación de nuevos conocimientos que ayuden a transformar sus entornos ${ }^{(1-4)}$.

Entre las diferentes estrategias para impulsar la investigación, destacan la formación, captación y retención de investigadores (5,6). En este escenario, la universidad se encarga de formar a los futuros investigadores a través del posgrado, y en el pregrado la investigación es uno de los ejes para la formación de futuros profesionales, particularmente de la carrera de medicina ${ }^{(7-12) .}$

En los países en desarrollo, como es el caso de los países sudamericanos, la producción científica médica es baja ${ }^{(13)}$, posiblemente debido a los limitados recursos económicos y humanos para la investigación ${ }^{(5)}$, a baja cultura de publicación ${ }^{(14)}$ y a deficiente formación en investigación (15). En Perú, los esfuerzos de las universidades para motivar a los estudiantes de Medicina en la investigación y la publicación son muy limitados ${ }^{(16,17)}$. Esto se refleja en ejemplos específicos relativos a la baja publicación ${ }^{(18-20)}$.

La investigación, como materia académica mas que un curso teórico se aprende haciendo, este proceso de aprendizaje debe incluir desde la idea de investigación hasta la publicación de los resultados en una revista científica (21). Dado que los docentes de cursos de investigación deben guiar al estudiante en dicho desarrollo, estos deberían haber realizado investigación previamente, es decir, han debido publicar artículos originales en revistas indizadas. No hemos encontrado ningún artículo que explore la frecuencia de publicación de docentes universitarios de cursos de investigación.

Por ello, el objetivo del estudio es conocer la frecuencia y los factores asociados a la publicación de artículos en revistas indizadas por los docentes universitarios de las escuelas de Medicina del Perú durante el año 2011.

\section{MATERIALES Y MÉTODOS}

\section{DISEÑO Y POBLACIÓN DE ESTUDIO}

Se realizó un estudio transversal analítico. Se incluyó a todos los docentes universitarios que figuraban en los sílabos de los cursos de investigación de pregrado (Metodología de la Investigación y Tesis), realizados el 2011 en las 32 escuelas de medicina del Perú. Los planes de estudios y programas de estudio de los cursos de investigación se buscaron a través de las sociedades científicas de estudiantes de Medicina locales ${ }^{(22)}$ durante el período de junio a noviembre de 2012.

\section{ESTRATEGIA DE BÚSQUEDA}

La búsqueda se realizó en tres bases de datos: Google Scholar, Scopus y Medline (23) entre abril y junio de 2013. Para detectar las publicaciones de los docentes universitarios, se utilizó todas las posibles combinaciones de los nombres y apellidos (paterno y paterno-materno), adicionando las palabras Perú o de la ciudad donde se encuentra la universidad en cada una de las bases de datos, esta estrategia es una variante de la usada en estudios previos para detectar la publicación de resúmenes de congresos o la producción de investigadores ${ }^{(14,24)}$. Se revisó cada artículo individualmente para evitar la inclusión de homónimos.

La búsqueda de las publicaciones de los docentes de cada universidad se hizo por duplicado, por investigadores sin relación con la universidad evaluada, el nivel de concordancia encontrado fue de $92 \%$. Uno de los investigadores estuvo a cargo del control de calidad mediante la revisión de cada uno de los expedientes, para resolver los casos discordantes.

\section{ARTÍCULOS PUBLICADOS}

Se consideró como "publicación" a todo artículo divulgado en una revista científica, se excluyó los libros, informes técnicos, tesis, posters o resúmenes en congresos científicos, y se clasificó como "original" si presentaba las secciones: introducción, métodos, resultados y discusión, o sus equivalentes. Se diferenció por el periodo de publicación y base de datos donde estaba disponible.

Se registraron todos los artículos publicados hasta el año 2011. Los resultados de la búsqueda fueron estratificados en tres periodos y según base de datos. De acuerdo con esto, la variable "publicación" se consideró positiva si hubieran publicado al menos un artículo: i) Por lo menos una vez en su vida (hasta el 2011); ii) en los últimos cinco años (2007-2011), y iii) en los últimos dos años (2010-2011).

Para cuantificar el número total de artículos, si los sujetos del estudio fueron los autores corresponsales y si habían publicado en revistas peruanas o no, solo se usó la base de datos SCOPUS. 


\section{VARIABLES DE LOS DOCENTES UNIVERSITARIOS}

El nombre del docente universitario se usó para identificar su edad y profesión en bases de datos públicas. La edad se midió usando la fecha de nacimiento que aparece en el seguro social de salud de cada docente (www. essalud.org.pe) y luego fue categorizada en menos de 40, 40-59 y 60 o más años. Con el sistema "conoce a tu médico" del Colegio Médico del Perú (www.cmp.org.pe) se identificó si eran médicos o no. El número de cursos que enseñan, y si eran los coordinadores del curso, se obtuvo a partir de los sílabos de cada curso.

\section{VARIABLES DE LAS UNIVERSIDADES}

En cuanto a la universidad, se categorizó según su fuente de financiamiento (pública o privada), su ubicación (Lima o provincias) y la obligatoriedad, de la tesis para obtener el título de médico (Sí o no). Para conocer la producción científica de cada universidad se realizó una búsqueda en SCOPUS en la colección de revistas de ciencias de la salud (> 6800 revistas, incluido todo Medline) incluyendo solo los artículos publicados durante el 2011; se ordenó las 32 universidades según su producción y se categorizó como universidad productiva a las que estaban en el quintil superior de producción científica ( $\geq 5$ artículos durante el 2011).

\section{ANÁLISIS ESTADÍSTICO}

Se generaron dos bases de datos para cada universidad en el programa Microsoft Excel, que luego se combinaron, revisaron y corrigieron utilizando Stata v.11.0. Se realizó un análisis descriptivo de las variables categóricas mediante frecuencias absolutas y relativas. Las variables numéricas se describieron mediante la media y desviación estándar o la mediana y rango intercuartílico, de acuerdo a la evaluación previa del supuesto de normalidad mediante la prueba de Shapiro Wilk. La asociación de las características individuales de los docentes y de sus universidades se evaluó según el haber publicado al menos un artículo original en Medline en los tres períodos considerados. Se calcularon las razones de prevalencias crudas (RP) y ajustadas (RPa), mediante regresión de Poisson simple y múltiple con varianza robusta, con sus respectivos intervalos de confianza al 95\% (IC 95\%). Las variables significativas en el análisis bivariado fueron incluidas en el modelo ajustado. Se consideraron significativos los valores $p<0,05$.

\section{CONSIDERACIONES ÉTICAS}

El estudio fue revisado y aprobado por el Comité de Ética del Hospital Nacional Docente Madre-Niño San Bartolomé, en Lima, Perú. Después de recolectar los datos de cada docente y universidad, los nombres fueron eliminados de la base de datos, con lo cual quedó una lista anónima antes del análisis.

\section{RESULTADOS}

Se incluyeron 201 docentes universitarios de los cursos de investigación impartidos en las 32 escuelas de Medicina del Perú durante el 2011; la mediana de docentes de investigación por universidad fue de 4 con un rango intercuartílico de 2 a 7 . El 76,1\% fueron varones y la edad media fue de $52 \pm 11$ años, el $87,1 \%$ de ellos fueron médicos. Las características detalladas de los sujetos del estudio se describen en la Tabla 1.

El $56,2 \%$ de los docentes publicaron un artículo en una revista científica al menos una vez en su vida, a través de la búsqueda de Google Scholar. El 30,9\% fueron

Tabla 1. Características de los docentes universitarios de cursos de investigación en las escuelas de medicina del Perú, 2011

\begin{tabular}{|c|c|c|}
\hline Características & $\mathbf{N}$ & $(\%)$ \\
\hline \multicolumn{3}{|l|}{ Sexo } \\
\hline Masculino & 153 & $(76,1)$ \\
\hline Femenino & 48 & $(23,9)$ \\
\hline \multicolumn{3}{|l|}{ Edad } \\
\hline$<40$ años & 29 & $(14,4)$ \\
\hline 40 a 59 años & 121 & $(60,2)$ \\
\hline$\geq 60$ años & 51 & $(25,4)$ \\
\hline \multicolumn{3}{|l|}{ Profesión } \\
\hline Médico & 175 & $(87,1)$ \\
\hline No médico & 26 & $(12,9)$ \\
\hline \multicolumn{3}{|l|}{ Coordinador de curso } \\
\hline Sí & 54 & $(26,9)$ \\
\hline No & 147 & $(73,1)$ \\
\hline \multicolumn{3}{|l|}{ Cursos que dicta } \\
\hline 1 curso & 140 & $(69,7)$ \\
\hline 2 cursos & 51 & $(25,4)$ \\
\hline 3 o más & 10 & $(4,9)$ \\
\hline \multicolumn{3}{|l|}{ Universidad (lugar) } \\
\hline Lima & 93 & $(46,3)$ \\
\hline Provincias & 104 & $(53,7)$ \\
\hline \multicolumn{3}{|l|}{ Universidad (financiamiento) } \\
\hline Público & 108 & $(53,7)$ \\
\hline Privado & 93 & $(46,3)$ \\
\hline \multicolumn{3}{|l|}{ Tesis obligatoria } \\
\hline Sí & 107 & $(53,2)$ \\
\hline No & 94 & $(46,7)$ \\
\hline \multicolumn{3}{|l|}{ Universidad productiva* } \\
\hline Sí & 84 & $(41,8)$ \\
\hline No & 117 & $(58,2)$ \\
\hline
\end{tabular}

* Quintil superior de universidades peruanas según su producción científica en revistas de ciencias de la salud indizadas a Scopus durante el 2011. 

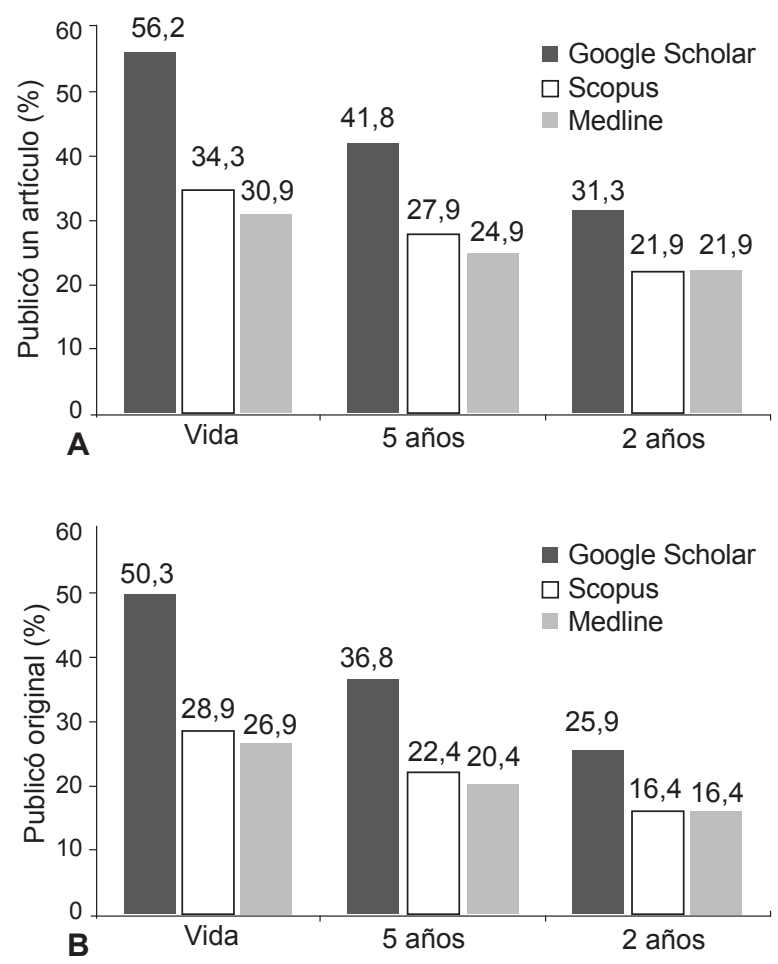

Figura 1. Publicación de artículos totales (A) y originales (B) por docentes de cursos de investigación de escuelas de Medicina del Perú, según tiempo y base de datos autores de una publicación en Medline. El 21,9\% los docentes universitarios habían publicado en Medline en los dos últimos años (Figura 1A).

De acuerdo a la búsqueda realizada en Google Scholar, el 50,3\% había publicado un artículo original por lo menos una vez y el $25,9 \%$ lo había hecho en los últimos dos años; 26,9 y $16,4 \%$ fueron las frecuencias equivalentes para Medline en los mismos períodos (Figura 1B).

Un tercio $(34,3 \%)$ de los docentes universitarios había publicado al menos una vez en una revista indizada en SCOPUS y el $23,8 \%$ lo había hecho en revistas no peruanas. El 10,9\% había sido autor corresponsal de un artículo, el 8,0\% fue autor de artículos originales y solo $3,0 \%$ había sido autor corresponsal de un artículo original en una revista no peruana indizada en Scopus.

En el análisis crudo, el tener menos de 40 años de edad, ser el coordinador de uno de los cursos de investigación, ser profesor en una universidad de Lima, ser profesor de una universidad privada, ser profesor de una universidad que tiene la tesis como la modalidad obligatoria para la graduación y el ser de una universidad productiva fueron los factores asociados con haber publicado un artículo en Medline en los tres períodos evaluados (Tabla 2). Aunque, el ser coordinador del curso no fue

Tabla 2. Factores asociados con la publicación de artículos originales en Medline según temporalidad en docentes universitarios de cursos de investigación de las escuelas de Medicina del Perú

\begin{tabular}{|c|c|c|c|c|c|c|}
\hline Publicación en Medline & RP crudo* & (IC 95\%) & $p$ & RP ajustado** & (IC 95\%) & $p$ \\
\hline \multicolumn{7}{|l|}{ Alguna vez en su vida } \\
\hline Tesis obligatoria & 2,77 & $(1,58-4,85)$ & $<0,001$ & 3,47 & $(1,47-8,21)$ & 0,005 \\
\hline Universidad productiva & 4,39 & $(2,51-7,68)$ & $<0,001$ & 3,12 & $(1,29-7,61)$ & 0,012 \\
\hline$<40$ años $^{\dagger}$ & 3,51 & $(1,71-7,21)$ & 0,001 & 2,15 & $(1,06-4,35)$ & 0,033 \\
\hline Universidad en Lima & 3,02 & $(1,78-5,12)$ & $<0,001$ & 1,59 & $(0,50-5,10)$ & 0,432 \\
\hline Coordinador de cursos & 0,47 & $(0,24-0,94)$ & 0,032 & 0,88 & $(0,49-1,60)$ & 0,686 \\
\hline Universidad privada & 1,82 & $(1,14-2,93)$ & 0,013 & 0,60 & $(0,26-1,42)$ & 0,249 \\
\hline \multicolumn{7}{|l|}{ En los últimos 5 años } \\
\hline Tesis obligatoria & 3,62 & $(1,76-7,47)$ & $<0,001$ & 6,00 & $(1,88-19,12)$ & 0,002 \\
\hline Universidad productiva & 6,27 & $(2,70-14,53)$ & $<0,001$ & 4,34 & $(1,57-12,02)$ & 0,005 \\
\hline$<40$ años $^{\dagger}$ & 4,40 & $(1,91-10,10)$ & $<0,001$ & 2,40 & $(1,11-5,17)$ & 0,026 \\
\hline Universidad en Lima & 4,13 & $(2,08-8,21)$ & $<0,001$ & 2,05 & $(0,53-7,93)$ & 0,298 \\
\hline Coordinador de cursos & 0,38 & $(0,16-0,92)$ & 0,031 & 0,81 & $(0,41-1,62)$ & 0,565 \\
\hline Universidad privada & 2,24 & $(1,25-4,02)$ & 0,007 & 0,43 & $(0,13-1,40)$ & 0,16 \\
\hline \multicolumn{7}{|l|}{ En los últimos 2 años } \\
\hline Tesis obligatoria & 3,95 & $(1,70-9,18)$ & 0,001 & 8,84 & $(2,60-30,12)$ & $<0,001$ \\
\hline Universidad productiva & 6,77 & $(3,15-14,55)$ & $<0,001$ & 3,24 & $(1,03-10,20)$ & 0,044 \\
\hline$<40$ años $^{\dagger}$ & 5,28 & $(2,13-13,05)$ & $<0,001$ & 2,97 & $(1,21-7,32)$ & 0,018 \\
\hline Universidad en Lima & 4,31 & $(1,96-9,50)$ & $<0,001$ & 3,29 & $(0,63-17,26)$ & 0,159 \\
\hline Coordinador de cursos & 0,49 & $(0,20-1,20)$ & 0,117 & 1,04 & $(0,52-7,32)$ & 0,921 \\
\hline Universidad privada & 2,32 & $(1,19-4,54)$ & 0,014 & 0,29 & $(0,08-1,09)$ & 0,067 \\
\hline
\end{tabular}


un factor asociado al publicar en los últimos dos años. El sexo, la profesión o el número de cursos impartidos no mostraron asociación significativa en el análisis bivariado en cualquier período.

En el modelo ajustado, ser menor de 40 años de edad, ser profesor en una universidad en la que se requiere una tesis para titularse y ser un profesor de una universidad productiva tuvo una asociación positiva con haber publicado en los tres períodos de tiempo considerados (Tabla 2).

\section{DISCUSIÓN}

La producción científica es una forma tangible y objetiva de medir la experiencia científica y la competencia en investigación, y es un indicador valioso en el proceso de acreditación de la universidades ${ }^{(3,25,26)}$.

Por lo tanto, sería deseable que los docentes universitarios de investigación estén realmente calificados en este campo. Sin embargo, lo encontrado fue prácticamente lo opuesto, solo la mitad de los docentes de investigación habían publicado al menos una vez en su vida, y los docentes de tres universidades fueron los únicos autores corresponsales de «artículos originales» en revistas no peruanas indizadas en Scopus. Se ha reportado previamente que dentro de las limitaciones para publicar se encuentra la falta de apoyo docente y la falta de experiencia de estos en publicación de artículos ${ }^{(27,28)}$, aspecto que es concordante con nuestros hallazgos.

Se reconoce que haber publicado o no, no garantiza que un docente de investigación tenga las competencias necesarias para enseñar. Sin embargo, el no haber publicado condiciona que un docente no pueda enseñar el proceso completo de investigación, que incluye la publicación de resultados en una revista científica ${ }^{(29)}$. En otros campos del conocimiento no se acepta que alguien que no conozca del tema lo enseñe, por ejemplo, que un médico general enseñe cirugía cardiovascular; de la misma forma, alguien que no ha publicado una investigación no debería "enseñar" investigación.

Los docentes que han publicado tienen mayor probabilidad de trabajar en universidades donde la tesis es la única modalidad para graduarse. Esto puede suponer que estas universidades tienden a apoyar más las investigaciones y, como resultado, se aseguran de contratar a docentes universitarios que publican, o puede ser el reflejo de los estudiantes y su interés por publicar sus tesis con sus asesores como coautores. Los cambios recientes en la Ley Universitaria peruana ${ }^{(30)}$ que implementa la tesis como única modalidad para obtener el título profesional, plantea un reto para todas las universidades, que deben pensar en tener un equipo adecuado de docentes que puedan asesorar tesis, quienes idealmente deben tener experiencia en publicación científica.

Asimismo, se encontró que los docentes de universidades productivas publican más que los de otras universidades (40,2\% frente a $8,6 \%)$. Esto puede deberse a que las universidades con un elevado número de investigadores tienen más opciones para elegir sus docentes de investigación, como es el caso de la Universidad Peruana Cayetano Heredia (UPCH), principal institución de investigación del país ${ }^{(20)}$. Al ser este un estudio transversal no se puede determinar si la universidad es productiva y si por ello captan docentes que investigan, o porque tienen docentes que investigan entonces su productividad es mayor, en todo caso, esta situación es un circulo virtuoso que permite incrementar la producción científica de la universidad. Por otro lado, las universidades productivas tienen un entorno más favorable para la investigación al crear más oportunidades para que los estudiantes participen, además, tienen más opciones para buscar tutores que los guíen en el proceso de investigación, aspecto importante en el proceso de formación y aprendizaje de la investigación ${ }^{(31,32)}$.

Los docentes más jóvenes fueron quienes más publicaron, este hallazgo también ha sido reportado en una universidad peruana ${ }^{(32)}$. Es posible que este grupo encuentre más fácil publicar por la familiaridad con las nuevas tecnologías que usan las revistas indizadas para el envío de artículos, así mismo, puede ser que su cultura de publicación sea diferente al tener acceso a más fuentes para publicar de las que habían en décadas previas, o el entusiasmo por producir sea mayor. Cualquiera que sea la explicación, las universidades deben tener en cuenta este aspecto cuando decidan contratar nuevos profesores de investigación.

La asociación encontrada en el análisis bivariado, de ser docente de una universidad ubicada en Lima y de pertenecer a una universidad privada, perdieron su asociación en el análisis ajustado. Esto se puede explicar por el efecto que generaron de dos instituciones, la UPCH y la Universidad Peruana de Ciencias Aplicadas, que son las universidades con mayor producción científica y la que más ha crecido en producción en los últimos años ${ }^{(33)}$, respectivamente.

El sexo del docente no fue un factor asociado con haber publicado. Este hallazgo no es concordante con los informes internacionales, en los que las mujeres tienen una producción científica más baja y limitada 
participación en investigación ${ }^{(34,35)}$. Ser el coordinador de un curso no fue un factor asociado, que muestra la deficiencia potencial en los pilares que sustentan la organización de los cursos de dichas universidades.

El estudio tiene limitaciones. En primer lugar, la evaluación de la producción científica de un investigador, además de la publicación en revistas científicas, incluye las patentes, fondos de investigación recibidos e impacto de las publicaciones a través de las citas; sin embargo, para llegar a este nivel de análisis se requiere una población con mayor actividad (36); en este caso, la mayoría no tenía publicaciones, o eran muy escasas, por lo que este análisis no habría mostrado un mensaje diferente. En este contexto, otra limitación es que se usó la publicación en forma dicotómica (sí/no publicó) y no por el número de artículos publicados, ya que no es lo mismo publicar una sola vez (investigador efímero) que alguien que publica en forma frecuente, pero por el escaso volumen de publicaciones fue mejor hacer el análisis presentado.

No se presentan los modelos para factores asociados con la publicación en Scholar Google y Scopus, se decidió por Medline por ser la base más usada y reconocida en el entorno médico peruano, y se consideró que los resultados son más entendibles, a pesar de que a partir de los datos de Scopus se generan los rankings de universidades iberoamericanas ${ }^{(33)}$. Por último, no se ha medido la capacidad pedagógica ni las habilidades docentes de los profesores, ya que se hubiese requerido una metodología diferente, que no fue objetivo del estudio.

En conclusión, la mitad de docentes universitarios de investigación de escuelas de Medicina del Perú nunca han publicado un artículo en una revista, tres de cada cuatro nunca han publicado un original en una revista indizada en Medline y solo uno de cada seis lo hicieron en los dos últimos años. Los factores asociados con haber publicado un artículo original en Medline fueron el tener menos de 40 años, ser profesor de una universidad en la que la tesis es obligatoria para titularse y ser un profesor de una universidad productiva científicamente.

Se recomienda que se capacite o renueve el personal docente de los cursos de investigación dictados en las escuelas de Medicina del Perú; que se incluya como criterio de selección y de mantenimiento en la cátedra el publicar artículos originales en revistas indizadas. De esta forma, los estudiantes de Medicina podrán formarse con personas que han culminado el proceso de investigación y tienen la experiencia suficiente para guiarlos desde la idea hasta la publicación de sus resultados. Esto ayudará a incrementar la producción científica de las escuelas de Medicina y de las universidades peruanas. Este reto es aun mayor para las universidades de provincia donde hay un escaso volumen de investigadores disponibles y su producción científica es muy baja ${ }^{(37,38)}$.

Contribuciones de autoría: PMT tuvo la idea de investigación y diseñó el estudio, CRM, RPE, ATR y JHR recopilaron los datos, PMT y CRM realizaron el análisis e interpretación de datos, todos los autores participaron en la redacción del manuscrito y aprobaron la versión final a publicar. Conflictos de interés: PMT estudió el pregrado en la
Universidad Nacional Mayor de San Marcos y el posgrado en
la Universidad Peruana Cayetano Heredia. PMT y CRM son
docentes de la Universidad Peruana de Ciencias Aplicadas,
donde RPE es alumno de pregrado. CRM estudió el pregrado en
la Universidad Ricardo Palma y el posgrado en la Universidad
Peruana Cayetano Heredia. JJHR y ATR son alumnos de la
Universidad San Martín de Porres. Ninguno de los autores
evaluó una universidad con la que tuvieran vinculación.

Financiamiento: autofinanciado.

\section{REFERENCIAS BIBLIOGRÁFICAS}

1. Ynalvez MA, Shrum WM. Professional networks, scientific collaboration, and publication productivity in resourceconstrained research institutions in a developing country. Res Policy. 2011 Mar;40(2):204-16.

2. Pavesi G, Siccardi A, Viale G, Grazioli C, Calciolari T, Tenchini ML, et al. Hedgehogs, humans and high-school science. The benefits of involving highschool students in university research. EMBO Rep. 2008 Mar;9(3):208-11.

3. Coccia M. A scientometric model for the assessment of scientific research performance within public institutes. Scientometrics. 2005;65(3):307-21.

4. Chabes A, Murillo JP, Huicho L, Castañeda M, Seclen S. Diagnóstico de la investigación biomédica en el Perú. Comunicación preliminar. An Fac Med. 1997;58(3):199-209.

5. Yagui M, Espinoza M, Caballero P, Castilla T, Garro G, Yamaguchi LP, et al. Avances y retos en la construcción del sistema nacional de investigación en salud en el Perú. Rev Peru Med Exp Salud Publica. 2010 JulSet;27(3):387-97.
6. Lansang MA, Dennis R. Building capacity in health research in developing world. Bull World Health Organ. 2004 Oct;82(10):764-70.

7. Provenzano AM, Graber LK, Elansary M, Khoshnood K, Rastegar A, Barry M. Short-term global health research projects by US medical students: ethical challenges for partnerships. Am J Trop Med Hyg. 2010 Aug;83(2):211-4. doi: 10.4269/ ajtmh.2010.09-0692.

8. Hren D, Lukic IK, Marusic A, Vodopivec I, Vujaklija A, Hrabak M, et al. Teaching research methodology in medical 
schools: students' attitudes towards and knowledge about science. Med Educ. 2004 Jan;38(1):81-6.

9. Detsky ME, Detsky AS. Encouraging medical students to do research and write papers. CMAJ. 2007 Jun;176(12):1719-21.

10. Vujaklija A, Hren D, Sambunjak D, Vodopivec I, Ivanis A, Marusic A, et al. Can teaching research methodology influence student' attitude toward science? Cohort study and nonrandomized trial in a single medical school. J Investig Med. 2010 Feb;58(2):282-6. doi: 10.231/ JIM.0b013e3181cb42d9.

11. Metcalfe D. Involving medical students in research. J R Soc Med. 2008 Mar;101(3):102-3. doi: 10.1258/ jrsm.2008.070393.

12. Venkatraman A, Nagpal SJ. Research opportunities for medical students in India. Natl Med J India. 2008 JulAug;21(4):202-3.

13. Huamaní C, González AG, Curioso WH, Pacheco-Romero J. Redes de colaboración y producción científica sudamericana en medicina clínica, ISI Current Contents 2000-2009. Rev Med Chil. 2012 Apr;140(4):466-75. doi: 10.4067/S003498872012000400007.

14. Toro-Polo LM, Pereyra-Elias R, NizamaVia A, Ng-Sueng L, Velez-Segovia E, Galan-Rodas E, et al. Publicación de los trabajos presentados a los congresos científicos de estudiantes de medicina, Perú 2002-2009: características y factores asociados. Rev Peru Med Exp Salud Publica. 2012 Oct-Dec;29(4):461-8.

15. Mayta-Tristán P, Cartagena-Klein R, Pereyra-Elías R, Portillo A, RodríguezMorales AJ. Apreciación de estudiantes de Medicina latinoamericanos sobre la capacitación universitaria en investigación científica. Rev Med Chil. 2013 Jun;141(6):716-22. doi: 10.4067/S003498872013000600005 .

16. Curioso WH, Lazo-Escalante M, Gotuzzo E, García HH, Gilman RH, García PJ. Entrenando a la nueva generación de estudiantes en salud global en una universidad peruana. Rev Peru Med Exp Salud Publica. 2008 Jul-Set;25(3):269-73.

17. Cabrera-Enríquez JA, Cruzado-Mendoza C, Purizaca-Rosillo N, López-Samanamú RO, Lajo-Aurazo Y, Peña-Sánchez ER, et al. Factores asociados con el nivel de conocimientos y la actitud hacia la investigación en estudiantes de medicina en Perú, 2011. Rev Panam Salud Publica. 2013 Mar;33(3):166-73.
18. Alarcón-Villaverde J, Romaní F, Gutiérrez C. Publicaciones científicas estudiantiles producidas en el curso de Epidemiología de la Facultad de Medicina de la Universidad Nacional Mayor de San Marcos durante el periodo 2003-2009. An Fac Med. 2010 Abr-Jun;71(2):111-6.

19. Arriola-Quiroz I, Curioso WH, CruzEncarnacion M, Gayoso O. Characteristics and publication patterns of theses from a Peruvian medical school. Health Info Libr J. 2010 Jun;27(2):148-54. doi: 10.1111/j.1471-1842.2010.00878.x.

20. Huamaní C, Mayta-Tristán P. Producción científica peruana en medicina y redes de colaboración, análisis del Science Citation Index 2000-2009. Rev Peru Med Exp Salud Publica. 2010 Set;27(3):315-25.

21. Mayta-Tristán P. Enseñando a publicar desde el pregrado. Rev Med Risaralda.2013;19(1):2-3

22. Pereyra-Elias R, Rodriguez-Morales AJ, Mayta-Tristan P. Undergraduate publication in Latin America: role of Medical' Scientific Societies. Med Teach. 2011;33(7):594.

23. Falagas ME, Pitsouni EI, Malietzis GA, Pappas G. Comparison of Pubmed, Scopus, Web of Science, and Google Scholar: strengths and weaknesses. FASEB J. 2008 Feb;22(2):338-42.

24. Mayta-Tristan P, Pereyra-Elias R, Mejia CR. Producción científica de los miembros vitalicios de la Academia Nacional de Investigadores Médicos. Rev Peru Med Exp Salud Publica. 2013 Oct-Dic;30(4):720-2.

25. Buela-Casal G. Reflexiones sobre el sistema de acreditación del profesorado funcionario de Universidad en España. Psicothema. 2007;19(3):473-82.

26. Carreras J. Evaluación de la calidad docente y promoción del profesorado (VI). Legislación universitaria española (d): modificación de la Ley Orgánica de Universidades. Profesorado funcionario: de la habilitación a la acreditación (2004-2008) (2a parte). Educ Med. 2009;12(3):131-47.

27. Molina-Ordoñez J, Huamani C, MaytaTristán P. Apreciación estudiantil sobre la capacitación universitaria en investigación: estudio preliminar. Rev Peru Med Exp Salud Publica. 2008;25(3):325-9.

28. Gutierrez C, Mayta P. Publicación desde el pregrado en Latinoamérica: importancia, limitaciones y alternativas de solución. CIMEL. 2003;8(1):54-60.

29. Gaught AM, Cleveland CA, Hill JJ 3rd. Publish or perish?: physician research productivity during residency training. Am J Phys Med Rehabil. 2013 Aug;92(8):710-4. doi: 10.1097/ PHM.0b013e3182876197.

30. Perú, Congreso de la República. Ley 30220: Ley Universitaria. Lima: Congreso de la República; 2014.

31. Prunuske AJ, Wilson J, Walls M, Clarke B. Experiences of mentors training underrepresented undergraduates in the research laboratory. CBE Life Sci Educ. 2013 Fall;12(3):403-9. doi: 10.1187/ cbe.13-02-0043.

32. Sogi C, Perales A, Anderson A, Bravo E. El proceso de producción científica de los investigadores de la Facultad de Medicina, UNMSM. An Fac Med. 2002;63(2):3140.

33. Scimago Research Group. Ranking de producción científica de universidades en Scopus: Perú 2014. Granada: Scimago Lab; 2014.

34. McCarren M, Goldman S. Research leadership and investigators: gender distribution in the federal government. Am J Med. 2012 Aug;125(8):811-6. doi: 10.1016/j.amjmed.2012.03.006.

35. Christianson M, Alex L, Wiklund AF, Hammarström A, Lundman B. Sex and gender traps and springboards: a focus group study among gender researchers in medicine and health sciences. Health Care Women Int. 2012;33(8):739-55. doi: 10.1080/07399332.2011.645970.

36. Vilchez-Roman C. Bibliometric factors associated with h-index of Peruvian researchers with publication indexed on Web of Science and Scopus databases. TransInformação. 2014;26(2):143-54.

37. Arroyo-Hernandez CH, De la Cruz W, Miranda-Soberon UE. Dificultades para el desarrollo de investigaciones en pregrado en una universidad pública de provincia, Perú. Rev Peru Med Exp Salud Publica. 2008 Oct-Dic;25(4):448-9.

38. Arroyo-Hernandez $\mathrm{CH}$, Zukeran-Medina EB, Miranda-Soberon UE. Características de la producción científica biomédica en Ica, Perú 1998-2007. Rev Peru Med Exp Salud Publica. 2009 Abr-Jun;26(2):203-6.

Correspondencia: Percy Mayta Tristán

Dirección: Escuela de Medicina de la

Universidad Peruana de Ciencias Aplicadas,

Lima, Perú.

Av. Brasil 2169 Dpto. 802, Jesús Maria,

Lima11, Lima, Perú.

Teléfono: 51987531133

Correoelectrónico:percy.mayta@upc.edu.pe 